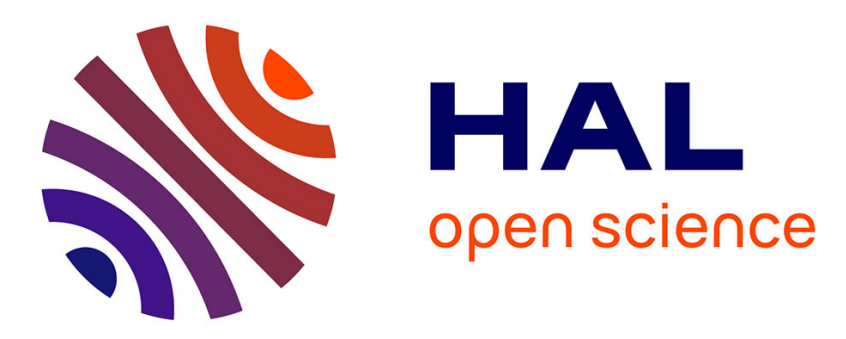

\title{
Instabilities of a liquid layer locally heated on its free surface
}

E. Favre, L. Blumenfeld, F. Daviaud

\section{To cite this version:}

E. Favre, L. Blumenfeld, F. Daviaud. Instabilities of a liquid layer locally heated on its free surface. Physics of Fluids, 1997, 9, pp.1473-1475. 10.1063/1.869470 . cea-01373966

\section{HAL Id: cea-01373966 https://hal-cea.archives-ouvertes.fr/cea-01373966}

Submitted on 29 Sep 2016

HAL is a multi-disciplinary open access archive for the deposit and dissemination of scientific research documents, whether they are published or not. The documents may come from teaching and research institutions in France or abroad, or from public or private research centers.
L'archive ouverte pluridisciplinaire HAL, est destinée au dépôt et à la diffusion de documents scientifiques de niveau recherche, publiés ou non, émanant des établissements d'enseignement et de recherche français ou étrangers, des laboratoires publics ou privés. 


\section{AIP $\left.\right|_{\substack{\text { Physics of } \\ \text { Fuids }}}$}

\section{Instabilities of a liquid layer locally heated on its free surface}

E. Favre, L. Blumenfeld, and F. Daviaud

Citation: Physics of Fluids 9, 1473 (1997); doi: 10.1063/1.869470

View online: http://dx.doi.org/10.1063/1.869470

View Table of Contents: http://scitation.aip.org/content/aip/journal/pof2/9/5?ver=pdfcov

Published by the AIP Publishing

\section{Articles you may be interested in}

Thermally excited fluid flow in a microsized liquid crystal channel with a free surface

Phys. Fluids 27, 062001 (2015); 10.1063/1.4921831

Stabilization of thin liquid films flowing over locally heated surfaces via substrate topography

Phys. Fluids 22, 042106 (2010); 10.1063/1.3407645

Linear stability of a volatile liquid film flowing over a locally heated surface

Phys. Fluids 21, 022105 (2009); 10.1063/1.3068757

Three-dimensional instability of a liquid layer flowing down a heated vertical cylinder

Phys. Fluids 12, 2198 (2000); 10.1063/1.1286594

Instabilities in a laterally heated liquid layer

Phys. Fluids 12, 1044 (2000); 10.1063/1.870359

\section{Looking for a specific} instrument?

Easy access to the latest equipment. Shop the Physics Today Buyer's Guide.

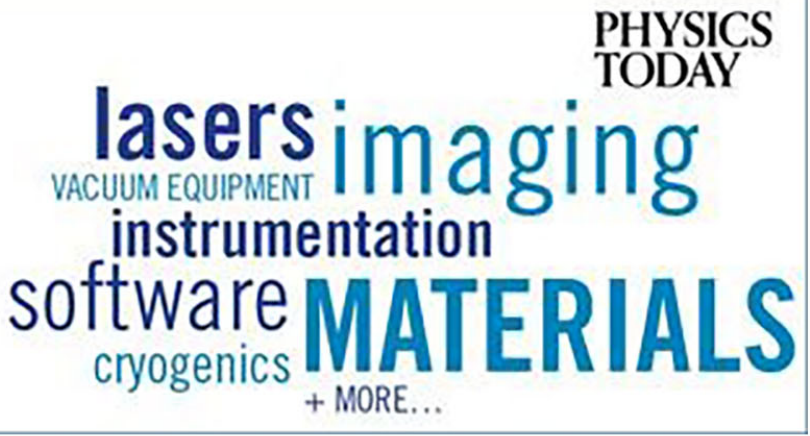




\title{
Instabilities of a liquid layer locally heated on its free surface
}

\author{
E. Favre and L. Blumenfeld \\ DCC/DPE/SPEA, CEA Saclay, F-91191 Gif-sur-Yvette Cedex, France \\ F. Daviaud \\ DSM/DRECAM/SPEC, CEA Saclay, F-91191 Gif-sur-Yvette Cedex, France
}

(Received 25 April 1996; accepted 16 January 1997)

\begin{abstract}
We report experimental results concerning patterns in a model experiment built to study buoyant-thermocapillary-driven flows. The fluid is situated in a cooled cylindrical container and locally heated on its free surface. The resulting temperature gradient induces a basic flow which draws the surface fluid from the hot center toward the cold boundary. When the gradient is increased and depending on the height of liquid, the basic flow destabilizes into different stationary patterns. Above a second threshold, the patterns become time-dependent. These different instabilities are characterized and compared to recent theoretical results. (c) 1997 American Institute of Physics. [S1070-6631(97)02105-3]
\end{abstract}

In the last decade, the behavior of buoyancy and thermocapillary-driven flows has received much attention both on fundamental and applied aspects. A linear stability analysis was carried out on thermocapillary flows, first without gravity, ${ }^{1,2}$ then in the presence of buoyancy. ${ }^{3-6}$ These works show that the basic flow becomes unstable toward longitudinal rolls or hydrothermal waves. Experiments have been performed on fluid layers with free surface subjected to a horizontal temperature gradient both in rectangular ${ }^{7,8}$ and cylindrical $^{9-11}$ containers. Some of the theoretical predictions have been recovered, such as the existence of hydrothermal waves, but many points still need to be clarified. This fundamental research is supported by the need for understanding the thermal and hydrodynamical properties of such flows in many applications such as floating zone crystal growth, ${ }^{12}$ electron-beam vaporization ${ }^{13}$ or laser welding. However, the real configurations are different from the theoretical ones. In particular, in the last two applications, the liquid is not driven by a horizontal thermal gradient on all its height but only on its surface. In this paper, we report new experimental results concerning the destabilization of a flow locally heated on its free surface. First, we describe the experimental set-up. Then we characterize the different instabilities which depend on the height of liquid and on the temperature difference. Finally, we discuss the experimental results and compare them with available theoretical studies.

The apparatus consists of a cylindrical container made of copper (diameter $2 R_{0}=10 \mathrm{~cm}$ and height $h_{0}=4 \mathrm{~cm}$; see Fig. $1)$. The bottom is made of sapphire to allow visualization. The container is thermally regulated by circulating water and a Plexiglass plate is situated a few millimeters above the free surface to reduce evaporation. It is filled with silicone oil (viscosity $\nu=0.0065$ Stokes) of Prandtl number $P=10$ to a height $h$ that is measured with a precision of $0.02 \mathrm{~mm}$. A meniscus is present at both boundaries. With the lid, $h$ remains constant to $1 \%$ over an experimental run. The liquid is locally heated on its surface by a cylindrical heater (diameter $2 r_{0}=3 \mathrm{~cm}$ ). The two parameters which control the experiment are $h$ and the temperature difference $\Delta T$ between the heater and the boundaries. The flow is characterized by temperature (thermocouples) and velocity (LDV) measurements and by shadowgraphy. The patterns are visualized by shadowgraphic imaging: A parallel vertical light beam crosses the container from bottom to top and forms a picture on a screen, due to surface deformations at the oil-air interface and to horizontal temperature gradients in the fluid. The spatio-temporal evolution of the structures is recorded using a video camera and the images are digitized along a circle to follow the azimuthal evolution of the patterns. ${ }^{8}$

The structure of the flow and the different observed patterns are shown on the stability diagram of Fig. 2. Each experiment is performed with $h$ fixed and $\Delta T$ variable. The thermocapillary and the buoyancy effect can be characterized by the Marangoni number $\mathrm{Ma}=-(\partial \sigma / \partial T) \Delta T h / \rho_{0} \kappa \nu$ and the Rayleigh number $\mathrm{Ra}=g \alpha \Delta T h^{3} / \kappa \nu$, where $g$ is the gravitational acceleration, $\alpha$ the thermal expansion coefficient, $\kappa$ the thermal diffusivity, $\rho_{0}$ the density of the fluid, $\nu$ the kinematic viscosity and $\sigma$ the surface tension.

Whatever the height of liquid $h$, a basic flow is present when $\Delta T$ is increased above zero (domain BF in Fig. 2). This flow is due to the thermocapillary effect and draws the surface fluid from the hot center towards the cold boundary. For large values of $h(h=15 \mathrm{~mm})$, Fig. 3 gives the radial velocity $V_{r}$ as a function of the depth $z$. The velocity is zero for $z<-8 \mathrm{~mm}$ and temperature measurements show horizontal isotherms in this zone. The thermocapillary effect creates a shear flow for $-3<z<0 \mathrm{~mm}$ and a return flow is present for $-8<h<-3 \mathrm{~mm}$. The basic flow thus appears as a torus and is in fact two dimensional (2D).

When $\Delta T$ is increased above a first threshold, whose value varies with $h$, the basic flow becomes unstable and stationary patterns are observed. Depending on $h$, three types of patterns are displayed. For small $h(h<2.8 \mathrm{~mm})$, a multiroll state made of co-rotating rolls is encircled by the basic flow and the resulting flow remains $2 \mathrm{D}$ [cf. domain $\mathrm{CR}$ in Fig. 2 and Fig. 4(a)]. These rolls are present near the heater. The number of rolls decreases with increasing $h$ ( 3 rolls for $h=2 \mathrm{~mm}$ ) and their wavelength is $\lambda \simeq 2.5 h$. For intermediate $h(3.2<h<5 \mathrm{~mm})$, the increase of $\Delta T$ leads to a pattern with stationary "petals" [cf. domain SP in Fig. 2 and Fig. 4(b)]. The radial extension of the petals is always less than $2 R_{0} / 3$ and their number decreases with $h$ from 14 for 


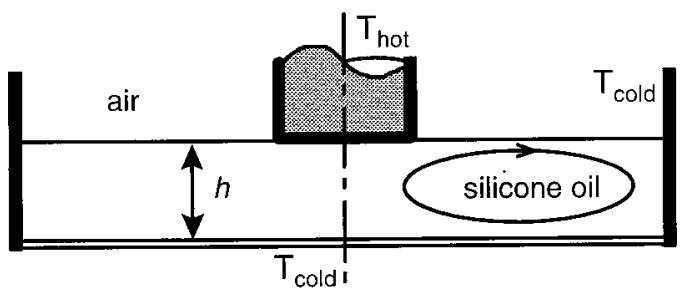

FIG. 1. Schematic drawing of the experimental apparatus.

$h=3.2 \mathrm{~mm}$ to 11 for $h=5 \mathrm{~mm}$. The azimuthal invariance is broken and the resulting flow is 3D. The transition from co-rotating rolls to petals displays intermediate structures near $h=3 \mathrm{~mm}$, where Ra Ma. For large $h(h>5.3 \mathrm{~mm})$, stationary radial structures appear [cf. domain SR in Fig. 2 and Fig. 4(c)]. For $h=10 \mathrm{~mm}$, the threshold corresponds to $\mathrm{Ma}=1.7 \times 10^{5}$ and $\mathrm{Ra}=2.1 \times 10^{6}$. Contrary to petals, the rays have a small azimuthal extension, they reach the cold vertical boundary and they do not extend over the whole depth. Their number decreases from 11 for $h=5.3 \mathrm{~mm}$ to 9 for $h=30 \mathrm{~mm}$. The rays correspond to counter-rotating rolls (with radial axis) which are superimposed on the basic flow, giving rise to a fully $3 \mathrm{D}$ state. The amplitude of the variation of $V_{r}$ increases with $\Delta T$ above the threshold. ${ }^{14}$

The patterns become time-dependent above a second threshold. For small $h$, azimuthal waves are observed very near the heater (cf. domain HW in Fig. 2). They consist of a source emitting a clockwise and an anti-clockwise rotating wave towards a sink. For $h=2 \mathrm{~mm}, 17$ rotating structures are evidenced at threshold and the associated frequency is $2 \nu / h^{2}$. The co-rotating rolls are slightly distorted by these waves. For higher $\Delta T$, radial pulsations are observed. For intermediate $h$, the stationary petals propagate azimuthally [cf. domain RP in Fig. 2 and Fig. 5(a)]. For $h=4.3 \mathrm{~mm}$, the azimuthal velocity of the pattern is $8.4 \nu / h$ at $r=19.3 \mathrm{~mm}$. For large $h$, the stationary rays oscillate azimuthally [cf. domain OR in Fig. 2 and Fig. 5(b)]. For $h=10 \mathrm{~mm}$, the threshold corresponds to $\mathrm{Ma}=4.0 \times 10^{5}$ and $\mathrm{Ra}=5.0 \times 10^{6}$. The frequency of oscillation $f_{0}$ increases with $\Delta T$. For $h=6.3$ $\mathrm{mm}$ and $\Delta T=11 \mathrm{~K}$ (resp. $h=15.2 \mathrm{~mm}$ and $\Delta T=7.5 \mathrm{~K}$ ) $f_{0}=2.9 \nu / h^{2}$ (resp. $f_{0}=14.6 \nu / h^{2}$ ). When the temperature difference is further increased, the dynamic becomes more complex and the system exhibits spatio-temporal chaos.

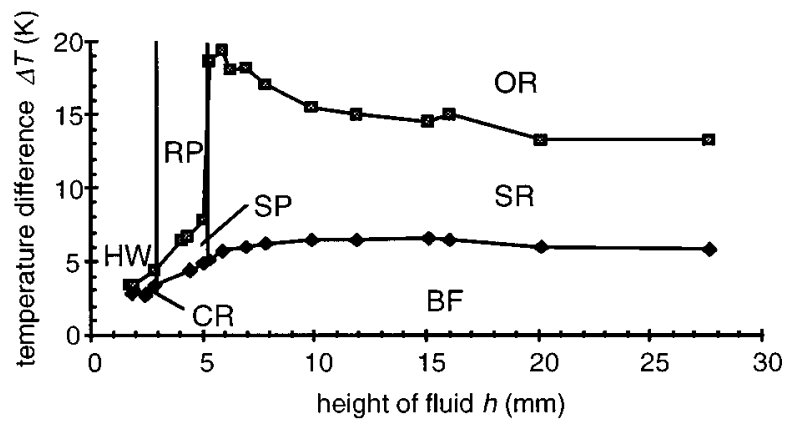

FIG. 2. Stability diagram: temperature difference $\Delta T_{c}$ vs height of liquid $h$. See text for details.

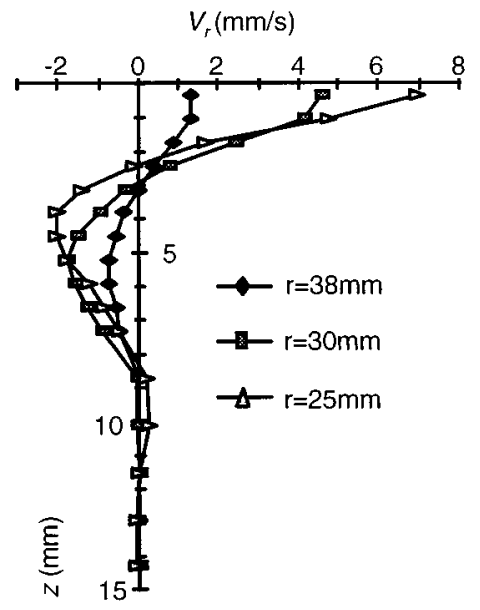

FIG. 3. Radial velocity profile at three different radii $r ; h=14.6 \mathrm{~mm}$ and $\Delta T=2.9 \mathrm{~K}$.

These results raise several interesting hydrodynamic issues. When $h$ is large, the basic toric flow develops only in the upper part of the liquid layer and for $h<-8 \mathrm{~mm}$, the fluid is at rest. This is due to a stable vertical stratification of the thermal field in the bottom of the cell. Experimental ${ }^{15}$ (resp. numerical ${ }^{11}$ ) results have displayed this stratification when $A=1$ (resp. $A \geqslant 0.75$ ). In our configuration, this fact becomes important when $A$ exceeds 0.3. Numerical simulations $^{3,7}$ and experiments ${ }^{7,9}$ for small $h$ and various $P$ have shown the multi-roll state, but the understanding of the origin of this state is still lacking. We interpret these rolls as being due to the dynamic interaction between the shear flow and the bottom. For typical critical values of $\Delta T$, the shear layer extends over the first $3 \mathrm{~mm}$ which exceeds the depth of fluid: The return flow of the basic torus cannot settle, hence the multi-rolls flow.

The rays correspond to rising hot fluid. This pattern appears when the height of liquid is quasi-infinite. However, the effect of the condition at the bottom is still present for $-10<h<-5 \mathrm{~mm}$, as shown by the reduction of their number (from 11 to 9 when $h$ increases) and by the decrease of the second critical curve of Fig. 2. For $h \geqslant 10 \mathrm{~mm}$ [i.e., for aspect ratios $\left.A=h /\left(R_{0}-r_{0}\right) \geqslant 0.3\right], \Delta T_{c}$ is independent of $h$. This result is in agreement with the results of Kamotani et al. $^{11}$ for $A>0.75$ and extends them. The rays could be related to an inversion of the vertical temperature profile near

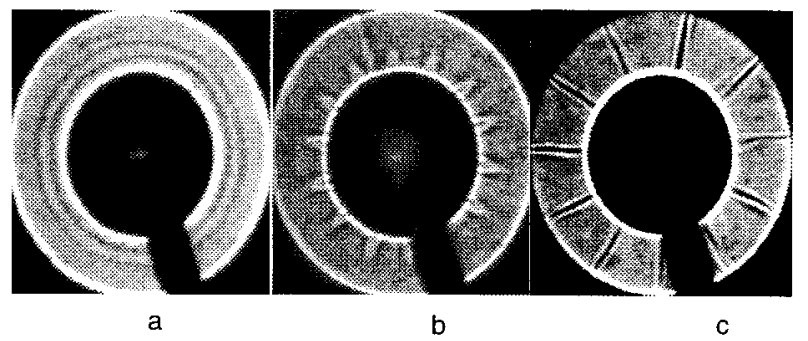

FIG. 4. Shadowgraphic images of the patterns in an horizontal plane. (a) for $h=1.8 \mathrm{~mm}$ and $\Delta T=3.2 \mathrm{~K}$; (b) for $h=4.3 \mathrm{~mm}$ and $\Delta T=6 \mathrm{~K}$, (c) for $h=15.0 \mathrm{~mm}$ and $\Delta T=10 \mathrm{~K}$. 

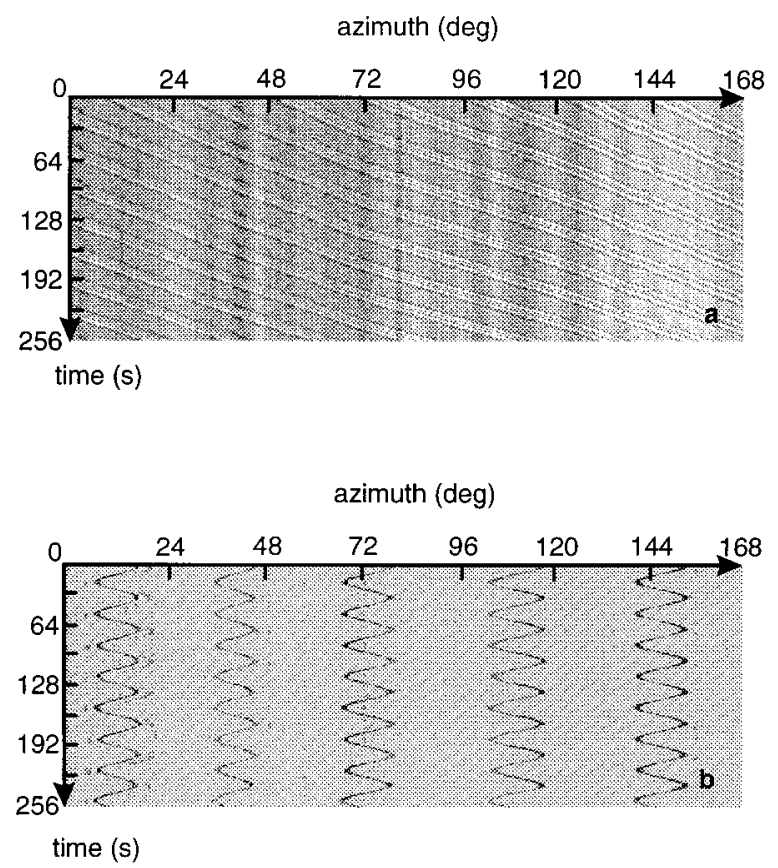

FIG. 5. Spatio-temporal evolution of (a) rotating petals $(h=4.3 \mathrm{~mm})$; (b) oscillating rays $(h=15.0 \mathrm{~mm})$.

the free surface and the cold wall, leading to a BénardMarangoni instability type, with a critical Marangoni number $\mathrm{Ma}_{c} \simeq 600$. This number is far greater than in the pure case where $\mathrm{Ma}_{c} \simeq 80$, because of the presence of the basic flow. Stationary rolls of Marangoni type have been found in some models, but associated with a linear flow ${ }^{1}$ or never critical. ${ }^{6}$ In the light of the interaction between the basic flow and the condition at the bottom, we propose the petals to be the pattern which develops when the bottom is situated in the extension of the upper part of the return flow (i.e., $-5<h<-3 \mathrm{~mm}$ ).

For small heigths, the dynamic states can be quantitatively compared to hydrothermal waves as defined in Refs. 1 and 6 and observed in Refs. 8 and 9. In fact, our results are closer to those of a recent study ${ }^{6}$ which takes into account the influence of thermal boundary conditions and geometry in the selection of the instability. The radial pulsations exhibited for higher $\Delta T$ are similar to those observed in Ref. 10 and could be due to a different instability. ${ }^{14}$ For larger heights, the observed time-dependent regimes do not correspond to available theoretical results and further work is needed. Let us note that the study of the hysteresis phenomenon of the transition to dynamic states for petals or rays reveals the subcritical nature of the bifurcation, whereas the primary instability is supercritical whenever $h$. The amplitude of the oscillations of the rays as well as the velocity of the petals increases with the distance from threshold.

In a liquid layer locally heated on its free surface, the basic 2D flow destabilizes into different patterns depending on the aspect ratio. These patterns have been experimentally characterized, as well as their transition to dynamical regimes. The structures observed at small height are similar to those observed in pure horizontal thermal gradient configurations both in experiments and theories. On the other hand, the patterns observed for larger heights appear to be new and fully $3 \mathrm{D}$. Work is under progress in this direction.

\section{ACKNOWLEDGMENTS}

We wish to thank N. Mukolobwiez, J. F. Mercier, and C. Normand for stimulating discussions, and M. Labouise and B. Ozenda for their technical assistance.

${ }^{1}$ M. K. Smith and S. H. Davis, "Instabilities of dynamic thermocapillary liquid layers," J. Fluid Mech. 132, 119 (1983).

${ }^{2}$ S. H. Davis, "Thermocapillary instabilities," Annu. Rev. Fluid Mech. 19, 403 (1987)

${ }^{3}$ H. Ben Hadid and B. Roux, "Buoyancy and thermocapillary-driven flows in differentially heated cavities for low Prandtl number fluids," J. Fluid Mech. 132, 119 (1983).

${ }^{4}$ G. Z. Gershuni, P. Laure, V. M. Myznikov, B. Roux, E. M. Zhukhovitsky, "On the stability of plane-parallel advective flows in long horizontal layers," Microgravity Q. 2, 141 (1992).

${ }^{5}$ P. Parmentier, V. Regnier, and G. Lebon, "Buoyant-thermocapillary instabilities in medium-Prandtl number fluid layers subject to a horizontal temperature gradient," Int. J. Heat Mass Transfer 36, 2417 (1993).

${ }^{6}$ J. F. Mercier and C. Normand, "Buoyant-thermocapillary instabilities of differentially heated liquid layers," Phys. Fluids 8, 1433 (1996).

${ }^{7}$ D. Villers and J. K. Platten, "Coupled buoyancy and Marangoni convection in acetone: experiments and comparison with numerical simulations," J. Fluid Mech. 234, 487 (1992).

${ }^{8}$ F. Daviaud and J. M. Vince, "Traveling waves in a fluid layer subjected to a horizontal temperature gradient," Phys. Rev. E 48, 4432 (1993).

${ }^{9}$ D. Schwabe, U. Moller, J. Schneider, and A. Scharmann, "Instabilities of shallow thermocapillary liquid layers," Phys. Fluids A 4, 2368 (1992).

${ }^{10}$ A. B. Ezersky, A. Garcimartin, J. Burguete, H. L. Mancini, and C. PerezGarcia, "Hydrothermal waves in Marangoni convection in a cylindrical container," Phys. Rev. E 47, 1126 (1993).

${ }^{11}$ Y. Kamotani, J. Masud, and A. Pline, "Oscillatory convection due to combined buoyancy and thermocapillarity," J. Therm. Phys. Heat Transfer 10, 102 (1996).

${ }^{12}$ J. J. Xu and S. H. Davis, "Convective thermocapillary instabilities in liquid bridges,” Phys. Fluids 27, 1102 (1984); F. Preissier, D. Schwabe, and A. Scharmann, "Steady and oscillatory thermocapillary convection in liquid columns with free cylindrical surface," J. Fluid Mech. 126, 545 (1983).

${ }^{13}$ S. Schiller, U. Heisig, and S. Panzer, Electron Beam Technology (Wiley, New York, 1982).

${ }^{14}$ E. Favre, L. Blumenfeld, and F. Daviaud, Phys. Rev. E (to be submitted).

${ }^{15}$ D. Schwabe, in Crystals (Springer Verlag, New York, 1988). 